\title{
ACUTE EPIGLOTTITIS COMPLICATED WITH PHARYNGEAL ABSCESS AND RECURRENT ACUTE AIRWAY OBSTRUCTION IN AN ADULT
}

\author{
*Mahesha Dabare \\ *Senior Registrar in anaesthesia and critical care, Base Hospital, Kanthale \\ *Corresponding author: mahesha.seneviratne@gmail.com
}

Key words: Acute epiglottitis, Acute airway obstruction, Deep neck abscess

\begin{abstract}
Acute epiglottitis is a common condition in children but is a rare entity in adults. Both in children and adults this is a life threatening condition as it can lead to complete airway obstruction. It is therefore vital that this condition is kept in mind when patients, both children and adults are admitted with severe airway obstruction to the emergency room. A case of adult acute epiglottitis complicated with abscess formation is described where timely intervention and correct diagnosis with appropriate treatment saved the life of the patient.
\end{abstract}

A 23-year-old, previously healthy man complained of a one day history of sore throat, odynophagia, dysphagia and fever. There was no evidence of immune-suppression from the past medical history. He did not have recent weight loss and his appetite was fair. There was no history suggestive of allergy, ingestion of foreign body or any intraoral or extra-oral trauma.

Physical examination revealed mild respiratory distress (respiratory rate: $22 / \mathrm{min}, \mathrm{SpO}_{2}: 90 \%$ on air). He was restless but the glasgow coma score (GCS) remained 15. He was more comfortable in the sitting position and was ill looking. Chest examination revealed few rhonchi and the air entry was reduced. The oropharynx was erythematous but no obvious oedema or asymmetry noted. Palpation of the neck revealed tender lymphadenopathy. His blood pressure was $110 / 70 \mathrm{~mm} \mathrm{Hg}$ and pulse rate was 110 beats per minute. He was admitted to the medical ward for observation. Initial resuscitation entailed oxygen via face mask, intravenous dexamethazone $8 \mathrm{mg}$ and nebulisation with salbutamol. Patient was connected to an electro cardiac monitor (ECG) and pulse and a pulse oxymeter. About one hour after admission the patient became restless with profuse sweating and developed a stridor. The saturation dropped to $93 \%$. He was brought to the intensive care unit (ICU).

On admission to the ICU patient was drowsy the $\mathrm{SpO}_{2}$ was $70 \%$ but it improved to $92 \%$ when supported with an ambu bag, tight seal mask and oropharyngeal airway with $100 \%$ oxygen. Securing the airway urgently was a necessity. The surgeon was summoned to be ready for emergency tracheostomy. A cricothyroidotomy puncture set was not available so it had to be improvised to use as the plan B. Midazolam 5mg was given and the ability to ventilate was established and suxamethonium 100mg was given. Significant oedema of the epiglottis and peri-laryngeal tissues was evident at laryngoscopy. A size $7 \mathrm{~mm}$ orotracheal tube was railroaded via a gum elastic bougie. He was paralysed and ventilated for a few hours before commencing SIMV mode. Further management of the patient was carried out as acute 
epiglottitis. Throat swab and blood culture was taken and intravenous cefotaxime $1 \mathrm{~g} 8$ hourly started. Dexamethazone and adrenaline nebulisation was also administered. The haemoglobin $(\mathrm{Hb})$ was $14 \mathrm{~g} / \mathrm{dl}$, white cell count (WCC) was $10,300 \mathrm{~mm}^{3}$ with a $91 \%$ neutrophil count. The chest $x$-ray antero-postero (AP) was normal. The erythrocyte sedimentation rate (ESR) was $18 \mathrm{~mm} /$ hour, and renal and hepatic function tests were normal. The $\mathrm{C}$-reactive protein (CRP) was elevated. Unfortunately the microbiological specimens were not processed at Trincomalee general hospital due to some technical problems. The fever settled within thirty six hours and the patient was extubated after 2 days when a leak around the deflated cuff was observed. There was a good clinical response and the patient was transferred to ward on the third day. Intravenous (IV) cefotaxime was continued until the $6^{\text {th }}$ day and he was discharged from the hospital with oral penicillin.

After 2 days the patient was readmitted to the emergency treatment unit (ETU) with stridor and respiratory distress. The airway was secured and he was sent to the ICU. The author was not directly involved with the intubation on this admission and it was done in the emergency treatment unit. Intubation was done with midazolam and suxamethonium. Patient was admitted to the ICU. IV cefotaxime, clarithromycin and metronidazole was commenced with dexamethazone. A flexible laryngoscope was not available so the pharynx was visualized with a paediatric gastroscope. There was significant swelling in the left pharyngeal wall and pyriform fossa. He was transferred to the teaching hospital Kurunegala for ENT opinion and drainage of abscess. Only an ulcer in the left pyriform fossa was noted on flexible fiber optic assessment at Kurunegala so the ENT surgeon concluded that probably the abscess would have ruptured during the journey. Patient was transferred back to Kanthale ICU and extubated after 3 days as planned. IV antibiotics were continued for 2 weeks. Naso gastric (NG) tube was kept in place for 6 days until he could tolerate oral feeding. The patient was discharged after 2 weeks with oral antibiotics to review in the clinic.

\section{Discussion}

Acute epiglottitis is a well recognized entity in children but is rare in adults. ${ }^{1}$ Acute epiglottitis in adults is often referred to as supraglottitis as the inflammation is generally not confined to the epiglottis but can also affect supraglottic structures such as the pharynx, uvula, base of the tongue, aryepiglottic folds or the false vocal cords. Overall mortality for adult epiglottitis is higher around $4-7 \%^{2,3}$ than in children $(2-3 \%)$ after the widespread immunization with HIB vaccine $^{4}$. However the mortality in adults further increases to $17 \%$ if complicated with airway obstruction.

The clinical presentation of the patient was typical of acute epiglottitis with all the common symptoms like odynophagia, inability to swallow secretions, sore throat, dyspnoea, fever and tachycardia1. The rapid deterioration to upper airway obstruction within 12 hours of onset, absence of obvious neck edema and visualization of a swollen epiglottis and perilaryngeal tissue led to the diagnosis of acute epiglottitis. Intubation always follows a diagnosis of epiglottitis in children but in adults it is controversial. Fiberoptic laryngoscopy is performed safely in adults in contrast to children and considered diagnostic ${ }^{3,6}$. The lateral chest $\mathrm{X}$ - ray showing oedematous epiglottis (the thumb sign) has a variable sensitivity. Diagnostic investigations are not a priority in an impending airway obstruction scenario.

Stridor occurs when more than $50 \%$ of the airway is narrowed. There is no conclusive evidence in the literature as to the best way of managing patients with upper airway obstruction. Some experts suggest inhalational induction and others claim that awake fiberoptic intubation is optimal ${ }^{5}$. Inability to achieve sufficient depth can be a disadvantage in inhalational induction but any episode of apnoea may allow the patient to lighten and resume spontaneous respiration which is the main advantage. Awake fiberoptic intubation is the other preferred choice and some studies quote a high success rate $(98.8 \%)$ in experienced hands ${ }^{5}$. The technique requires equipment and skill to perform. Blind nasal intubation is contraindicated in suspected airway abscess. It 
has infrequent success on the first pass and increased trauma with repeated attempts precipitating complete airway obstruction ${ }^{6}$. The risk of general anaesthesia and muscle relaxation is that if intubation is not successful, it may precipitate complete airway closure and make face mask ventilation impossible, thus necessitating an emergency airway ${ }^{7}$.In managing this patient the above risk was present but we established the ability to ventilate before administering suxamethonium. If such a situation came to light we were ready with plan $B$ to perform emergency needle cricothyroidotomy followed by emergency tracheostomy. Gas induction would have been a better option but the ICU was not equipped with an anaesthetic machine and it could have been time consuming to import one from the operating theatre (OT) in the limited time. We had neither the equipment nor the expertise for awake fiber optic intubation.

It is important to consider other differential diagnosis like deep neck abscesses (retropharyngeal, peripharyngeal and peritonsillar), tracheobronchitis, allergic reactions, foreign body aspiration and tumours ${ }^{7}$. Widening of the pre-vertebral space occur in retropharyngeal abscess. Computerised tomography (CT) is done only when the course of illness is prolonged or when the diagnosis is in doubt. Since the patient clinically improved we did not consider transferring him to another hospital for CT.

The treatment of acute epiglottis entails IV antibiotics, humidified oxygen, steroids and nebulised adrenaline. IV antibiotics should cover haemophilus influenzae, staphylococcus aureus, streptococcus and pneumococcus (amoxicillin/clavulanic acid or a third generation cephalosporin). Unlike in children in adults only $20 \%$ of epiglottitis is caused by haemophilus influenzae ${ }^{8}$. Regarding corticosteroid use, randomised controlled trials are lacking but the available evidence does not show significant benefits to reduce the need for intubation and shorten the clinical course ${ }^{9,10}$. Patients should be extubated once a leak is detected with a deflated cuff.
Management of deep neck abscesses involve high dose intravenous antibiotics and surgical drainage. If the size of the abscess is small and there are no imminent complications, a trial of conservative management may be attempted. In our patient the abscess ruptured spontaneously therefore surgical drainage was not necessary. Corticosteroids are added if there is no contraindication. The polymicrobial nature ( $\beta$-haemolytic Streptococcus, Staphylococcus aureus, Bacteroides and Neisseria spp) of the infection calls for broad spectrum cover ${ }^{11}$.

In the discussed case the seriousness of the clinical signs and symptoms were underestimated at the time of admission which led to the deterioration to acute airway obstruction. The patient was managed as acute epiglottitis in the ICU. He was discharged on oral penicillin from the medical ward before completing the full course of cefotaxime. A possible explanation for the recurrence of acute airway obstruction is worth discussing. Acute epiglottitis would have got complicated with suppuration or we would have misdiagnosed the first presentation. In retrospect, patient should have been discharged after adequate treatment with broad spectrum antibiotics, ENT assessment and fiberoptic laryngoscopy. Perhaps a CT scan would have revealed the true extent of the infection and an alternate course would have been pursued.

In summary, upper airway infections like epiglottitis and deep neck abscesses can be lifethreatening. Airway compromise and the potential for abscess rupture are ever-present. A thorough evaluation of the airway is an absolute necessity, and should include use of flexible fiberoptic laryngoscopy and CT scan when necessary. A careful airway plan should be established in the presence of a senior anaesthetist and ENT surgeon in a limited time 
frame which is challenging. Appropriate broad spectrum antibiotics should be given for an adequate period preferably with a microbiological diagnosis. Surgical drainage of abscess should not be delayed if significant and in the presence of airway obstruction.

\section{References}

1. Franziska Wick, Peter E. Ballmer, Alois Haller, Epiglottitis in adults, Swiss Medical Weekly, 2002;132:541-547

2. Fontanarosa PB, Polsky SS, Goldmann GE. Adult

epiglottitis. J Emerg Med 1989;7: 223-31.

3. Knöbber D, Bergbreiter R. Zur Klinik der akuten Epiglottitis Erwachsener. Laryngo-Rhino-Otol 1991;70: 695-7.

4. Mayo-Smith MF, Spinale JW, Donskey CJ, Yukawa M, Li RH, Schiffmann FJ. Acute epiglottitis:an 18-year old experience in Rhode Island.Chest 1995;108:1640-7.

5. Flavell E M, Stacy M R, Hall J E; The clinical management of airway obstruction, Current anaesthesia and Critical care;2009:1 20( 3), 102112

6. Parhiscar A, Har-El G. Deep neck abscess: a retrospective review of 210 cases. Ann Otol Rhinol Laryngol, 2001;110: 1051-4.

7. Irani BS, Martin-Hirsch D, Lannigan F. Infection of the neck spaces: a present day complication. $J$ Laryngol Otol 1992; 106:455-8.

8. Heeneman H, Ward K. Epiglottic abscess: Its occurrence and management. J Otolaryngol 1977;6:31-6.

9. Dort JC, Frohlich AM, Tate RB. Acute epiglottitis in adults: diagnosis and treatment in 43 patients. J Otolaryngol 1994; 23: 281-5.

10. Mayo-Smith MF, Hirsch PJ, Wodzinski SF, Schiffmann FJ. Acute epiglottitis in adults, an eight-year experience in the State of Rhode Island. N Engl J Med 1986;314: 133-9.

11. Alaani A, Griffiths H, Minhas S S, Olliff J, Drake Lee A B; Parapharyngeal abscess: diagnosis, complications and management in adults, Eur Archhives of Otolaryngology(2005);262:345-350

12. Donald J. Heindel, MD, Deep Neck Abscesses in Adults: Management of a Difficult Airway, Anaesthesia; 1987;66: 7766 